\title{
Coherence Lifetime broadened Optical Transitions in a 2 atom diameter HgTe nanowire: a temperature dependent Resonance Raman study.
}

\author{
Joseph H. Spencer ${ }^{a}$, David C. Smith ${ }^{a}$, Liam P. McDonnell ${ }^{a}$, Jeremy Sloan ${ }^{b}$ and Reza J. Kashtiban ${ }^{b}$ \\ a. School of Physics and Astronomy, University of Southampton, University Road, Southampton, Hampshire, SO161BJ. \\ b. Dept. Physics, University of Warwick, Coventry, CV47AL
}

This paper presents the results of measurements of the temperature dependence of resonance Raman scattering from 2 atom diameter HgTe nanowires embedded in the central pore of single walled carbon nanotubes, over the temperature range 4-295K and excitation photon energies in the range 1.65 to $1.90 \mathrm{eV}$. The spectra are analysed to extract the temperature dependence of the phonon energies and linewidths and the central energy, linewidth and peak scattering strength of the optical resonances. The temperature dependence of the peak scattering strength is explained by a model in which the resonance broadening is dominated by coherence lifetime broadening, allowing us to determine the coherence lifetime of the underlying optical transition: $9 \mathrm{fs}$ at $295 \mathrm{~K}$ and $18 \mathrm{fs}$ at $50 \mathrm{~K}$. The results are comparable with similar results on carbon nanotubes which suggests that the optical transitions responsible for the Raman resonances are excitonic.

\section{Introduction}

Quantum engineering in nanoscale structures with reduced dimensionality has led to a huge range of exciting science and technology. Varying the number of quantum confined dimensions to produce quantum wells, wires and dots leads to quite different physics and applications in each case. In quantum wires the combination of strong quantum confinement, charge transport, reduced screening and constrained scattering leads to effects such as Luttinger liquids, ${ }^{1}$ long distance ballistic electron transport, Majorana fermions ${ }^{2}$ and excitonic states in metallic systems ${ }^{3}$ amongst many other unique effects. One common theme within these effects is greater coherence of electronic excitations.

Whilst there has been a significant amount of research on quantum wires this has mostly been on structures with dimensions $>10 \mathrm{~nm}$, which corresponds to wires with cross-sections containing tens of thousands of atoms. ${ }^{4}$ More recently there has been a huge amount of research on single walled carbon nanotubes (SWCNTs) with diameters in the $1 \mathrm{~nm}$ range, which has led to the discovery of a range of new physics. ${ }^{5,6}$ Despite their small diameters SWCNTs are still in many ways far from the limit of the smallest possible quantum wires, e.g. atomic chains. For instance, their bandstructure can be fairly accurately derived from the bandstructure of graphene using an envelope approximation. ${ }^{7,8}$

Recently there is starting to be some exciting research on quantum wires approaching the extreme, atomic chain limit. For instance Majorana fermions have been observed in 2 by 1 atom wide ribbons of Fe atoms formed on Pb crystals. ${ }^{2}$ In another study 2 by 3 atom transition-metal dichalcogenide (TMD) wires have recently been formed from TMD monolayers by direct electron beam writing ${ }^{9}$. This work suggests that it might be possible to produce integrated devices based on extreme diameter nanowires. Using extreme nanowires produced by filling the inner pore of SWCNTs it has been shown that few atom diameter quantum wires often have different bonding to the bulk parent material, i.e. they are entirely new allotropes. ${ }^{6}$ Very recently filling of small diameter SWCNTs has been enabled the production of long atomic chains of metal halides. ${ }^{10}$ These studies on extreme diameter nanowires clearly indicate that there is still a lot of new quantum wire physics to be discovered particularly in wires of one to a few atoms in diameter.

In this paper we elucidate the nature of one of the optical transitions of 2 atom diameter HgTe nanowires formed by filling SWCNTs, by determining the temperature dependence of resonance Raman scattering of the nanowires phonons. We demonstrate that the Raman scattering intensity increases by more than an order of magnitude with decreasing temperature from $292 \mathrm{~K}$ to $4 \mathrm{~K}$, contrary to the phonon population which decreases by a factor of 4 . The temperature dependence of the Raman intensity of the HgTe nanowires indicates that the underlying optical transition's width is predominantly due to the coherence lifetime broadening.

\section{Experimental}

The experiments were performed on a sample prepared by filling of electric-arc produced SWNTs (see work by Krestinin et al. ${ }^{11}$ ) with HgTe using the melt infiltration technique. ${ }^{12-14}$ After the melt infiltration the samples were treated by gentle refluxing in 1:1 mixture of concentrated $\mathrm{HCl}$ and $\mathrm{HNO}_{2}$ for $1 \mathrm{hr}$, followed by washing in deionised water, followed by collection on a porous membrane (0.22 $\mu \mathrm{m}$ Millipore) in order to remove extraneous HgTe outside of 
the filled tubes. ${ }^{15}$ The samples were then fully characterised using HRTEM to ensure that no extraneous HgTe remained and determine the atomic structure of the filling structures. ${ }^{15,16}$ Inspection of more than 100 SWCNTs from the supplied sample by HRTEM revealed that the significant majority ( $85 \%)$ fall within a limited diameter range of $1.2-1.6 \mathrm{~nm}$ and that more than $50 \%$ of these SWCNTs were observed with filling. All of the nanotubes observed with filling within this diameter range were found to correspond to the novel tubular form of HgTe. This form differs from the bulk form presented in panel a of figure 1 . This new structure (panel b of figure 1), which has also has been observed in a range of other HgTe filled SWCNTs, ${ }^{17,18}$ has a unit cell consisting of $4 \mathrm{Hg}$ and $4 \mathrm{Te}$ atoms, belonging to the rod group $\# 29, \mathrm{p} 4_{2} / \mathrm{m}$, with point group symmetry $\mathrm{C}_{4 \mathrm{~h}}$ and a translation vector length along the chain axis of $0.446 \mathrm{~nm}$. The tubular form of $\mathrm{HgTe}$ is most stable within nanotubes of a diameter range between $1.35-1.45^{17,18}$ (fig 1 panel c). For Raman scattering measurements, cleaned, filled nanotube product was added to ethanol and sonicated for 20 mins in a bath sonicator, allowed to rest for $24 \mathrm{hrs}$ to allow any aerosolized tubes to settle, and then drop cast onto an oxide coated $(200 \mathrm{~nm})$ silicon substrate.

Resonance Raman spectra were measured for laser energies in the range 1.65 to $1.90 \mathrm{eV}$, and sample temperature in the range 4-295K using a triple spectrometer Raman system. The sample was mounted in a helium flow cryostat, and held under a moderate vacuum of $\sim 1 \mathrm{e}^{-6} \mathrm{mbar}$. The laser source was a continuous wave titanium sapphire laser (1.65 -1.82 eV) or a DCM dye laser (1.77- 1.9 $\mathrm{eV}$ ). The laser source was pre-filtered to remove any background fluorescence via a tuneable laser line filter or a volume Bragg grating. ${ }^{15}$ The sample was excited and the Raman scattering collected via an Olympus LMPLan-IR 50x objective with an NA of 0.55 yielding a laser spot size of $2 \mu \mathrm{m}$. The laser power dependence of the Raman scattering was determined and the spectra presented taken well within the low power linear regime with $0.1 \mathrm{~mW}$ incident at $4-150 \mathrm{~K}$ and below and $1 \mathrm{~mW}$ incident at $225-295 \mathrm{~K}$. The spectra presented have been corrected for the throughput of the spectrometer. The spectra presented are associated with three different combinations of excitation source and pre-filter associated with three excitation energy ranges. Differences in the optics associated with the three different combinations lead to slightly different signal magnitudes at the same excitation energy. This effect was corrected for by a single scaling factor associated with the two higher excitation energy ranges determined by using at least 4 measurements in each overlap region. Once scaled the results from the different systems agree to within the estimated experimental error. Particular care was taken to ensure the repeatability of the Raman scattering intensities ${ }^{15}$ leading to a repeatability of the magnitude of the scattering of $\sim 8 \%$ as determined from independent measurements at several excitation energies over the course of the whole experimental period. A detailed description of the experimental system and method can be found in Ref. ${ }^{15}$

\section{Results and discussion}

In figure 2 we present representative Raman spectra of the $\mathrm{HgTe}$ filled SWCNT sample measured with excitation photon energy $1.776 \mathrm{eV}$ at $4,100,200$ and $292 \mathrm{~K}$. The $4 \mathrm{~K}$ spectrum is in total agreement with those published in our previous publication on the 4K Raman spectra of HgTe filled SWNT samples. ${ }^{16}$ As set out in that publication there is strong evidence, including the polarisation dependence of the scattered light, resonance behaviour and DFT calculations, to support the assignment of the majority of the peaks to the vibrational modes of the 2 atom diameter HgTe wires shown in Fig 1 . The only Raman peaks also observed in Raman spectra from unfilled SWCNTs are two Radial Breathing mode peaks which form a doublet at $\sim 180 \mathrm{~cm}^{-1}$. All of the other peaks observed in the spectra can be attributed to the fundamental, harmonic and combination peaks of four vibrational modes of the HgTe wires; labelled A $\left(47 \mathrm{~cm}^{-1}\right), B\left(50 \mathrm{~cm}^{-1}\right), C\left(94 \mathrm{~cm}^{-1}\right)$ and $D\left(114 \mathrm{~cm}^{-1}\right)$. By comparing the new, higher temperature spectra with the $4 \mathrm{~K}$ spectrum it is obvious that there are no sudden changes, e.g. new peaks, in the spectra which would indicate a phase change. However there is a very significant reduction in the intensity of the Raman scattering associated with the HgTe phonon peaks with (a)

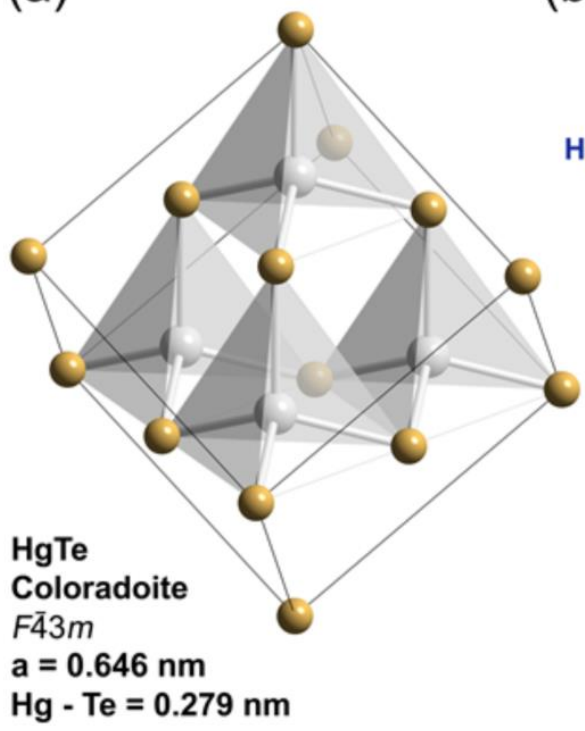

(b)

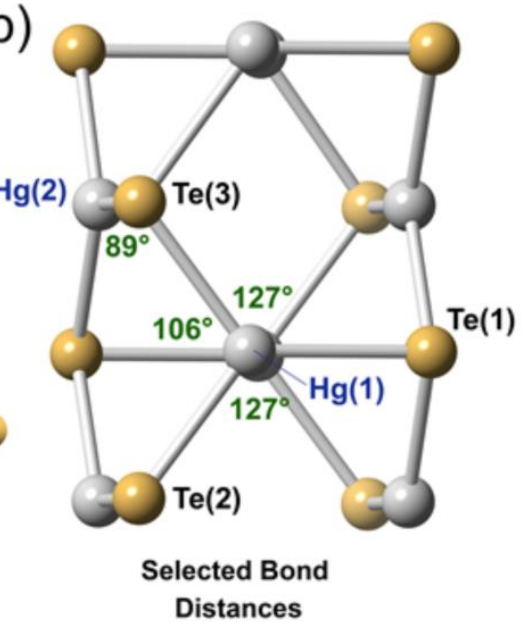

$\mathrm{Hg}(1)-\mathrm{Te}(1)=0.272 \mathrm{~nm}$ $\mathrm{Hg}(1)-\mathrm{Te}(2)=0.276 \mathrm{~nm}$ $\mathrm{Hg}(1)-\mathrm{Te}(3)=0.275 \mathrm{~nm}$ $\mathrm{Hg}(2)-\mathrm{Te}(3)=0.272 \mathrm{~nm}$ (c)

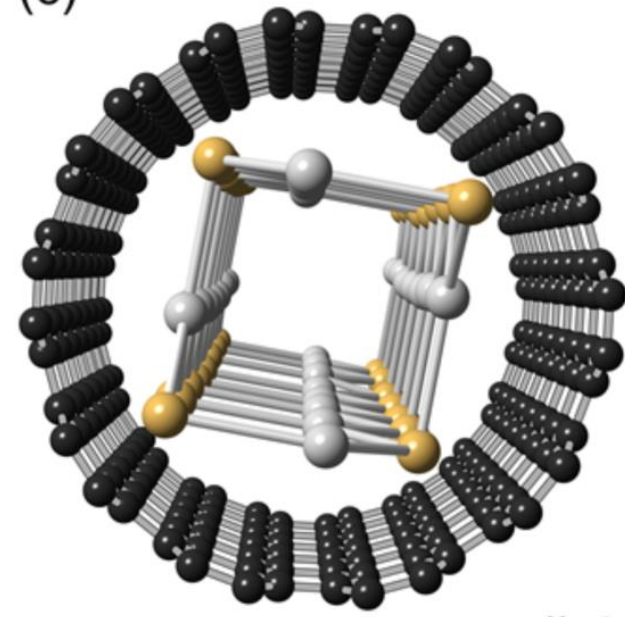

$\mathrm{Hg} O$

Figure 1: (a) Bulk zinc blend structure of $\mathrm{HgTe}$ with the $\mathrm{Hg}$ and Te positions reversed relative to the reported form ${ }^{30}$ and with the average $\mathrm{Hg}$-Te bond distance indicated. (b) Side on view of the DFT optimized 'tubular' form of HgTe (adapted from ${ }^{17}$ ). Te-Hg-Te bond angles in green (colour online version) and selected bond distances are indicated. (c) End-on view of the structure from (b) mounted in a $(10,10)$ conformation SWNT. 

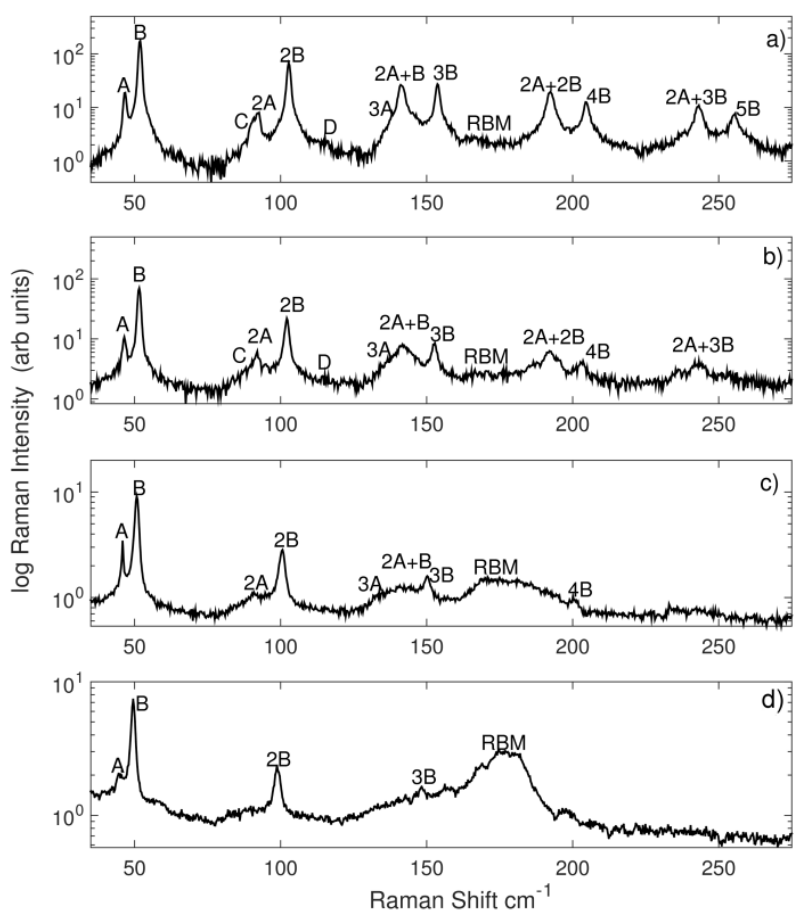

Figure 2 Multiple Raman spectra acquired at $1.776 \mathrm{eV}$ for sample temperatures of a) 4, b) 100 , c) 225 and d) $295 \mathrm{~K}$.

increasing temperature.

As set out in our previous work, at $4 \mathrm{~K}$ the Raman features associated with the HgTe nanowires are strongly resonant for excitation photon energies in the range $1.7-1.85 \mathrm{eV}$. Thus one
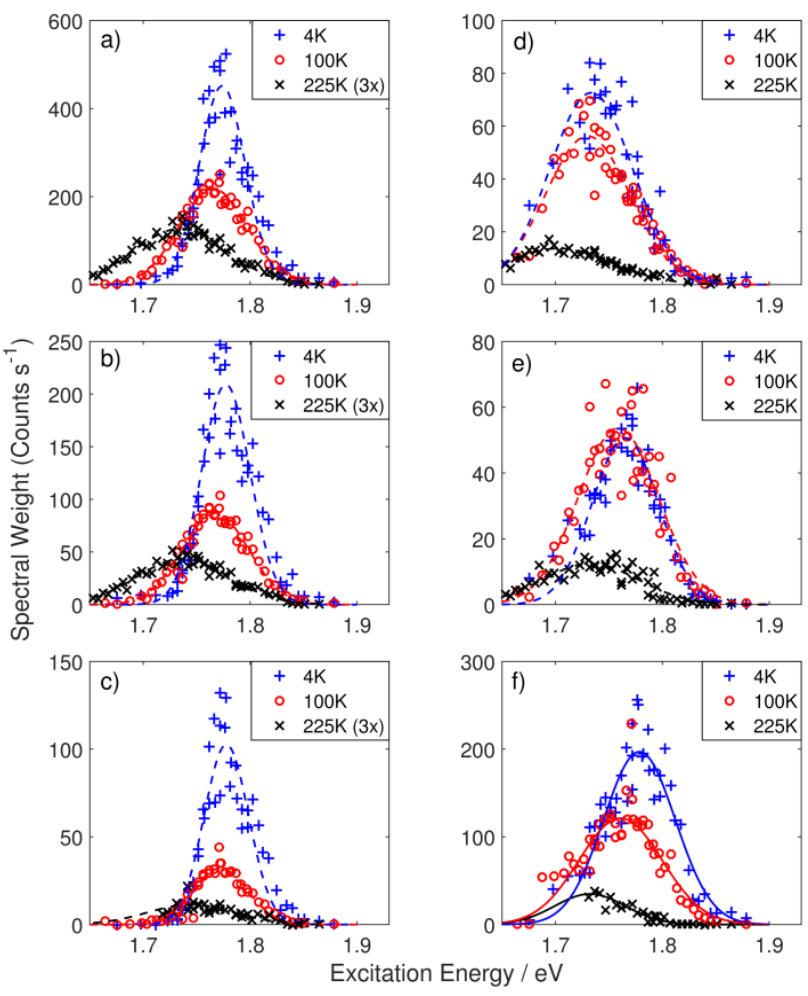

Figure 3 Evolution of spectral weight for the fitted modes $B, 2 B, 3 B, A, 2 A$ and $2 A+B$ in panels (a)-(f) respectively at 3 sample temperatures of $4 K, 100 K$ and $225 \mathrm{~K}$, represented by blue plus signs, red circles and black crosses. The $225 \mathrm{~K}$ data in panels (a)-(c) have been scaled by a factor of 3 for ease of viewing. possible cause of the strong temperature dependence of the intensity of the wire Raman scattering is a shift in the energy of the optical transition responsible for the resonance. To investigate this possibility the excitation photon energy dependence of the Raman spectra at 4, 25, 50, 100,170, 225 and $295 \mathrm{~K}$ were measured. The peaks observed in these spectra were then fitted using Lorentzian lineshapes to extract the centre shift, line width and spectral weight of the various peaks as a function of temperature and laser energy. None of the centre shifts or linewidths for any of the peaks show any significant laser energy dependence at any temperature. They do show some dependence on temperature however these dependencies are all explainable in terms of softening of the vibrational modes and enhanced anharmonic decay of the phonons with increased temperature; both common effects in crystalline systems. Due to their standard behaviour the centre shifts and linewidths are not reported in detail in the main paper but are reported in the supplementary material.

On the other hand the spectral weights of the peaks are strongly resonant with laser energy at all temperatures with the form of the resonance varying significantly with temperature. This can clearly be seen in figure 3 where the resonance curves for the $B, 2 B$ and $3 B, A, 2 A$ and $2 A+B$ peaks at $4 K, 100 K$ and $225 K$ are presented. The peaks were chosen for presentation as their resonance curves are the clearest however similar behaviour is seen for the other peaks (see supplementary material). It is clear from the data presented in figure 3 that the resonances red shift, broaden and significantly weaken with increasing temperature. As discussed in more detail later, it is not a priori possible to predict the shape of the resonance. Least squares fitting of a variety of standard lineshapes including Gaussian, Lorentzian and double Lorentzian were attempted but it was not possible to identify a clear best lineshape (see supplementary material). Therefore the resonances were all fitted using a Gaussian lineshape to allow quantification of the centre energy, width and amplitude of the resonance as a function of temperature. The results of these fits are presented in figures 4 ,

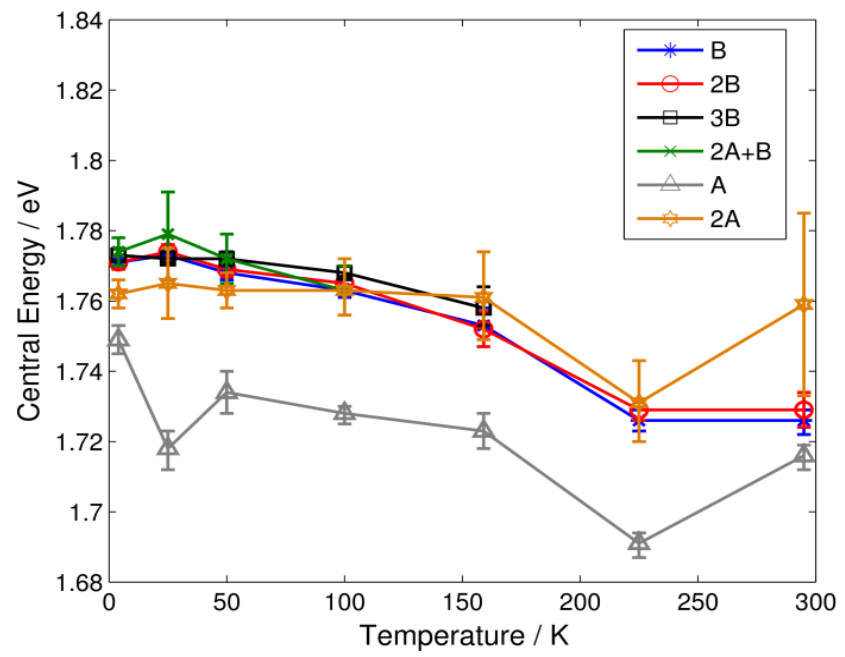

Figure 4 Temperature dependence of the fitted central energy for the resonances observed. Error bars correspond to the $95 \%$ confidence bands from the fitting. 
5 and 6.

In the data presented in figure 4 the $B, 2 B, 3 B$ and $2 A+B$ resonances have the same centre energy for their resonances. It is also clear that the resonances of the $A$ and $2 \mathrm{~A}$ peaks occur at lower energies with the $A$ resonance occurring at the lowest energy. In all cases the resonances slightly red shift with increasing temperature as is often seen in crystalline systems due to lattice expansion. The difference in central energy between the $A$ and $B$ modes is reasonably constant across most of the temperature range at $\approx 30 \mathrm{meV}$. This difference is significantly greater than the phonon energies and comparable with the resonance widths. The existence of combination peaks involving $A$ and $B$ modes means we can be confident that the optical transitions responsible for the two resonances are associated with the same nanowire and the fact that the resonances overlap suggests that the excitations responsible for the transitions are closely related.

The resonance widths presented in figure 5 also show a clear difference between the $A$ and $2 A$ peaks and the $B$ peak with the $A$ and $2 A$ resonances being significantly broader at low temperatures. The $2 A+B$ resonance width is more in line with the $A$ peaks than the $B$ peaks. In all cases the resonance widths are significantly greater than the phonon energies or linewidths and increase significantly with increasing temperature. Of the various contributions to the linewidth of optical transitions the one which is most commonly temperature dependent is the coherence linewidth. Thus it is likely that at higher temperatures the resonance linewidth is dominated by the coherence lifetime of the underlying optical transitions. This conclusion is strengthened by a detailed analysis of the temperature dependence of the spectral weight which forms the basis of the rest of the analysis in this paper.

The amplitude of the resonances of all the peaks shown in figure 6 strongly decrease with increasing temperature with the $A, B$ and $2 B$ peaks decreasing by factors of 11,10 and 20 from $4 \mathrm{~K}$ to $295 \mathrm{~K}$. These drops are even more dramatic when the fact that the phonon

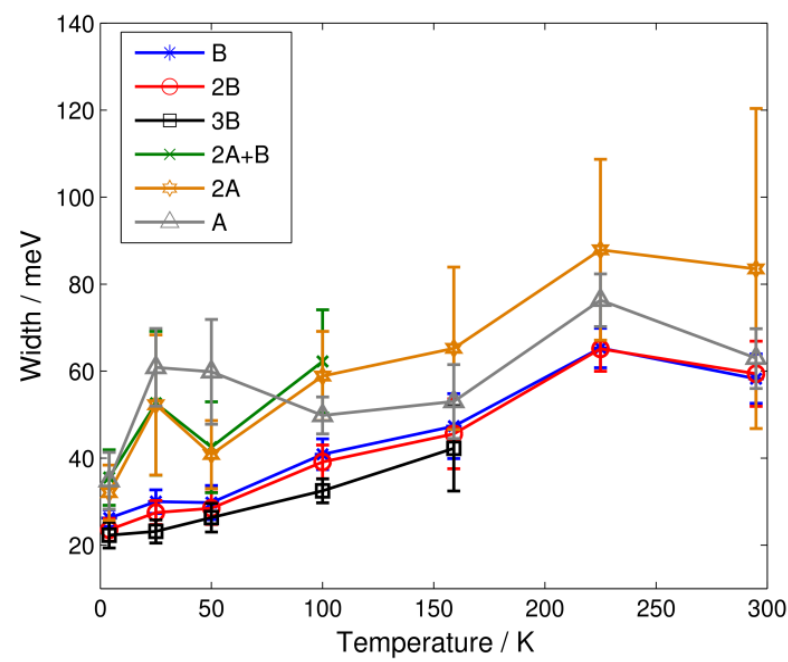

Figure 5 Evolution of spectral width for the fitted modes $B, 2 B, 3 B, A, 2 A$ and $2 A+B$ as a function of temperature. Error bars in the figure correspond to the $95 \%$ confidence bounds from the fitting.

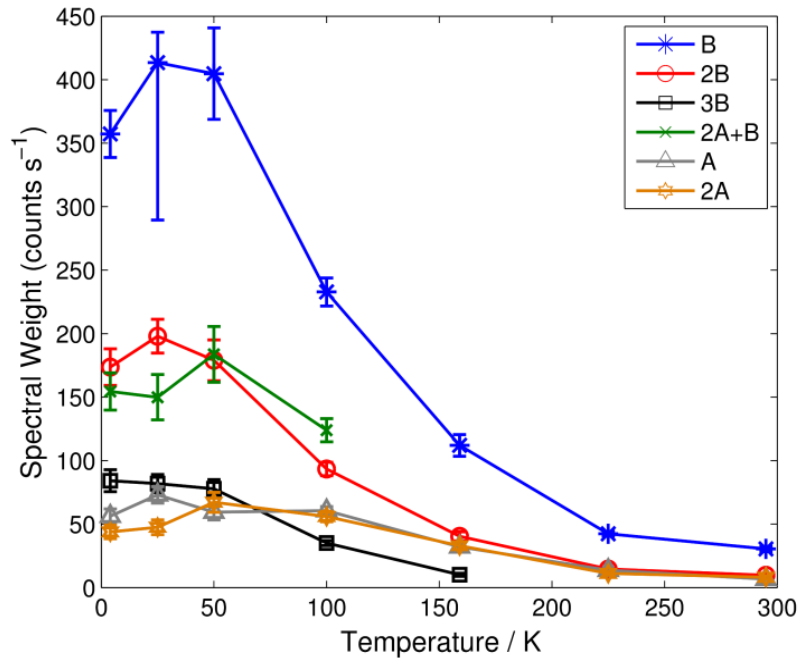

Figure 6 Fitted spectral weight of all measured Raman resonances as a function of temperature. Spectral weight was calculated by converting measured linewidth into a pixel value and multiplying the fitted amplitude of the resonance by this pixel width. Error bars correspond to the $95 \%$ confidence bands from fitting.

populations associated with the Raman peaks are expected to increase significantly over the same temperature range; at $295 \mathrm{~K}$ the thermal population will be 5 .

To understand this data we need to consider in detail the theory of Raman scattering. The validity of time-dependent perturbation theory calculations for predicting Raman scattering intensities is well established and the application of Feynman diagrams to these calculations is common. ${ }^{19}$ The Feynman diagram representing the leading order perturbation for single phonon scattering and the expression for the scattering intensity which comes from it for a homogeneously broadened system are presented in figure 7(a). The summation in the expression is over the optically available electronic excitations within the nanowires. In an inhomogenously broadened system, which is certainly a possibility in an ensemble of nanowires, this expression has to be averaged over the inhomogeneous distribution. Whilst the theoretical expression is relatively simple to derive the complexities of applying it to specific systems are well known. For instance predictions of resonance lineshapes require knowledge of the electronic density of states, the energy dependence of coherence lifetime of the electronic states and sample inhomogeneity. In the case of multi-phonon Raman scattering the situation is made even more complex by the possibility that the scattering can in principle occur via simultaneous or sequential emission of phonons. The Feynman diagrams and expressions for the scattering intensity for the two cases for two phonon emission are presented in figures $7(b)$ and 7 (c) respectively. 


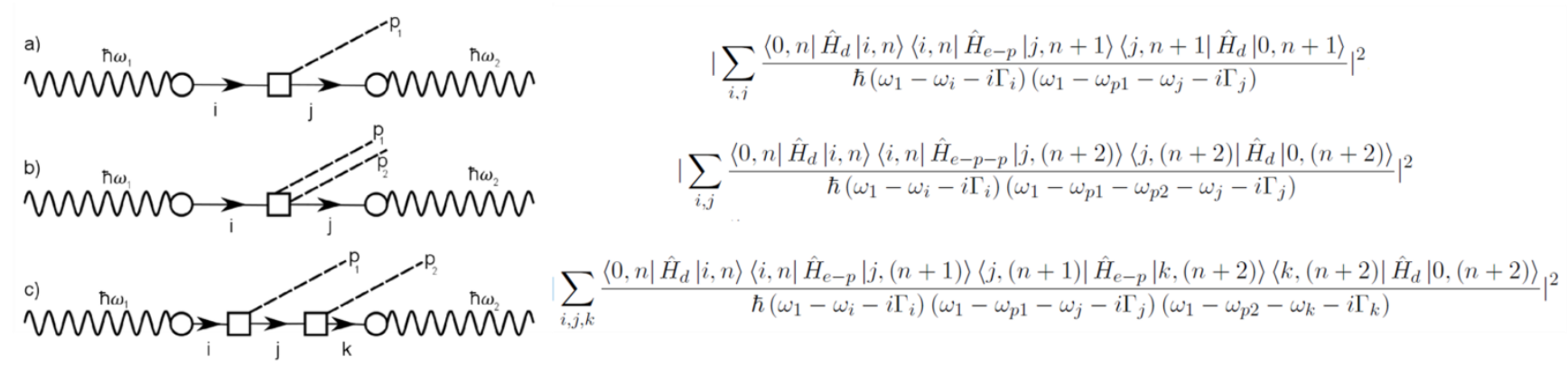

Figure 7 Feynman diagram for a) 1 phonon emission b) 2 phonon, simultaneous emission and c) 2 phonon, sequential emission. To the right is the corresponding equation for the probability of the Raman scattering.

The strongest effect present in the data in this paper is the temperature dependence of the scattering intensity. Within the time-dependent perturbation theory there are two obvious ways in which temperature affects the intensity of scattering; the phonon population dependence of the electron-phonon scattering matrix element and the dependence on electronic coherence where the coherence linewidth is likely to increase with temperature.

In the case of one phonon scattering the population contribution to the temperature dependence of the scattering intensity is clear and we can predict that the scattering intensity should follow $1+n$ where $\mathrm{n}$ is the phonon population. In the case of two phonon scattering the prediction of the population contribution depends on whether the phonon emission is into a single phonon mode, in which case the prediction is $(1+n)(2+n)$, or into many different phonon modes, in which case the prediction is $(1+n)^{2}$. The fact that the Raman resonance linewidth is two orders of magnitude larger than the phonon linewidth strongly suggests that the system is in the limit that we can treat phonon dephasing as negligible during the Raman scattering process. In this case the electron-phonon interaction leads to a coherent superposition of phonon modes which can be treated as a single phonon and thus the $(1+n)(2+n)$ predictions applies. By extension the prediction of the population contribution to the three phonon scattering intensity's temperature dependence is either $(1+n)^{3}$ in the multi-phonon limit or $(1+n)(2+n)(3+n)$ in the single-phonon limit with the latter being more likely.

In general the perturbation theory predicts that an increase in the resonance linewidth should be accompanied by a reduction in the scattering intensity at the peak of the resonance. However, the strength of the correlation depends on the relative importance of

\begin{tabular}{|c|c|c|c|c|c|c|}
\hline \multirow[b]{2}{*}{ Peak } & \multicolumn{3}{|c|}{ Single phonon correction } & \multicolumn{3}{|c|}{ Multi phonon correction } \\
\hline & $\begin{array}{l}\text { Lower } \\
95 \%\end{array}$ & Value & $\begin{array}{l}\text { Upper } \\
95 \%\end{array}$ & $\begin{array}{l}\text { Lower } \\
95 \%\end{array}$ & Value & $\begin{array}{l}\text { Upper } \\
95 \%\end{array}$ \\
\hline B & -6.0 & -4.6 & -3.1 & -6.0 & -4.6 & -3.1 \\
\hline $2 B$ & -8.0 & -6.2 & -4.3 & -8.7 & -6.7 & -4.7 \\
\hline $3 B$ & -10.4 & -7.1 & -3.7 & -12.4 & -8.7 & -4.9 \\
\hline
\end{tabular}

Table 1: Best fit slopes and their $95 \%$ confidence limits obtained from linear fits to the phonon population corrected spectral weight vs resonance linewidth data presented in figure 8 for the two phonon population correction scenarios discussed in the main text for the $\mathrm{B}, 2 \mathrm{~B}$ and $3 \mathrm{~B}$ peaks. the different contributions to the linewidth, i.e. on the relative importance of the electronic coherence lifetime, the width of any continuum of electronic states, and sample inhomogeneity. As already discussed the temperature dependence of the resonance linewidth supports the hypothesis that the electronic coherence lifetime dominates the resonance width at higher temperatures. If this is the case the theory predicts that the peak scattering intensity should follow Gamma ${ }^{-2(\mathrm{~N}+1)}$ where $\mathrm{N}$ is the number of intermediate electronic states involved in the process, i.e. Gamma ${ }^{-4}$ for one phonon or simultaneous multi-phonon scattering, Gamma ${ }^{-6}$ for two phonon sequential scattering and $\mathrm{Gamma}^{-8}$ for three phonon sequential scattering. If the optical transition is broadened by an underlying continuum of optical transitions then this would be expected to decrease the dependence on the coherence linewidth. In the limit that the linewidth was predominantly due to a continuum of states the coherence linewidth would to first order have no effect on the Raman scattering intensity at the peak of the resonance. However it is difficult to reconcile this limit with the clear temperature dependence of the Raman resonance's linewidth. If the temperature dependence of the linewidth was due to an increasing inhomogeneous broadening the Raman scattering intensity at the peak of the resonance should follow the inverse of the linewidth.

In order to explore the correlation between the resonance linewidth and intensity separate from any phonon population effect we present plots of the log of the spectral weight at the peak of the resonance corrected for the phonon population dependence versus the $\log$ of the $B$ resonance width for the $B, 2 B$ and $3 B$ peaks in figure 8. The (a) and (b) panels cover both of the phonon population scenarios already discussed. Also shown are best linear fits to the various data sets; the best fit slopes and their $95 \%$ confidence limits are presented in table 1.

The results of the linear fits indicate that the best explanation for the temperature dependence of the Raman scattering intensity is the model which assumes that the $\mathrm{B}, 2 \mathrm{~B}$ and $3 \mathrm{~B}$ resonances can be treated as being due to a single optical transition which is coherent lifetime broadened and that the phonon emission in the higher order harmonics occurs sequentially. It is clear that the results cannot be explained in terms of a temperature dependent inhomogeneous broadening and that the width of the transitions cannot be dominated by a continuum of optical transitions. 

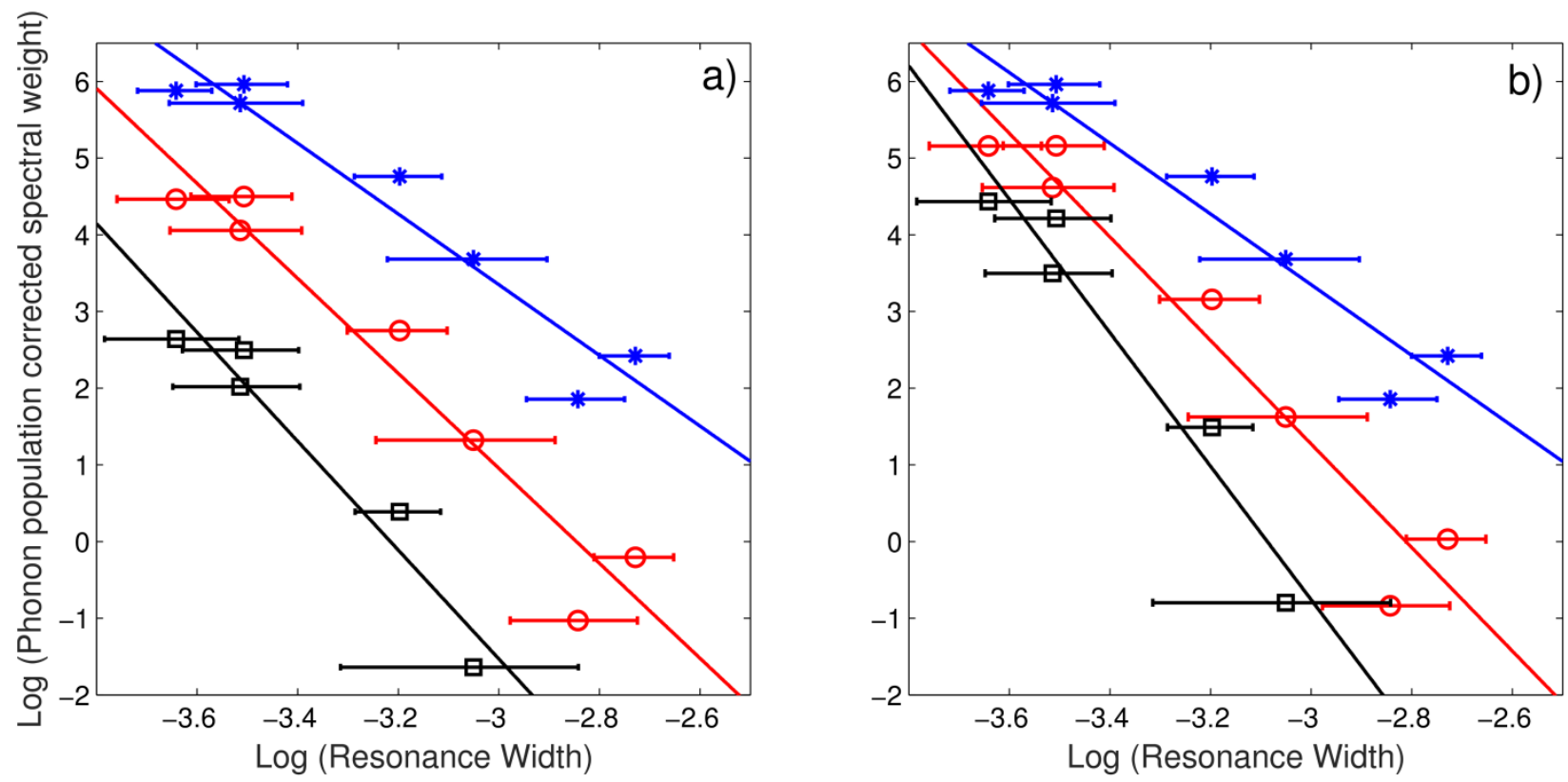

Figure $8 \mathrm{Log}$ of the phonon population corrected spectral weight as a function the changing resonance width of the $\mathrm{B}$ mode. Panel a) shows the phonon correction of $(1+n),(1+n)(2+n)$ and $(1+n)(2+n)(3+n)$ for the $B, 2 B$ and $3 B$ resonances. Panel $b)$ has been corrected for using $(1+n),(1+n)^{2}$ and $(1+n)^{3}$ for the $B, 2 B$ and $3 B$ respectively. In each panel of the figure the blue stars, red circles and black squares are the data for the $B, 2 B$ and $3 B$ resonances respectively.

However it is not entirely possible to exclude the possibility of simultaneous emission of phonons.

Another clear prediction of the perturbation theory is that in the limit that the optical transition is coherence lifetime limited and the phonon emission is sequential the resonance linewidth should decrease with phonon number. There is some evidence for this in the results presented in figure 5 . However the theory predicts that the ratio of the linewidths, rather than their absolute difference, should remain constant as the linewidths increase with increasing temperature and this is not seen. In addition attempts to fit the observed Resonance lineshapes assuming the optical transition width is coherence linewidth dominated and the phonon emission is sequential were not successful (see supplementary data for further details). These observations might be considered to favour the simultaneous emission model which predicts the same resonance width for the different harmonics. However another possible explanation is that there is something subtly wrong with the existing model. One possibility not yet included in the model is that in the case of multi-phonon Raman the intermediate states which are not involved in the optical transition may be dark states with non-zero momentum. In bulk semiconductors this mechanism is key to explaining the details of multi-phonon Raman. In SWCNTs, an archetypal 1D system, it is known ${ }^{20,21}$ that dark excitons play an important role in the optical properties.

Whether or not the phonon emission is simultaneous or sequential and involves or doesn't involve dark intermediate states, the results presented in figure 8 support the hypothesis that the optical transition responsible for the Raman resonance's width is dominated by coherence lifetime broadening over most of the temperature range down to at least 50K. If this is the case then it should be possible to determine the coherence lifetime from the linewidth. As the conversion factor for the harmonic peaks depends upon whether the phonon emission is simultaneous or sequential, we analyse the $B$ peak alone. In this case we obtain estimates of the coherent lifetime for the underlying optical transition of $9 \mathrm{fs}$ at $292 \mathrm{~K}$ and $18 \mathrm{fs}$ at $50 \mathrm{~K}$.

It is interesting to consider, what is the nature of the optical transitions that is responsible for the resonances? Whilst it is not possible to be entirely definitive, the most likely explanation is that the transitions are excitonic. Excitonic transitions, unlike band to band occur at discrete energies. In this case then it is likely that the two resonances associated with the $\mathrm{A}$ and $\mathrm{B}$ phonons are due to closely related excitonic excitations whose different symmetry leads to them coupling to one or other but not both of the $A$ and $B$ phonons. An obvious mechanism for this is if the two states are effectively fine structure of a "parent" exciton split because of a weak symmetry breaking perturbation. Further clarification of this issue will require theoretical predictions of the bandstructure which are not currently available.

It is interesting now that we have more fully analysed the HgTe resonance Raman behaviour to compare this with the published behaviour of the resonance Raman of single and double walled carbon nanotubes. Whilst there are a considerable number of papers $^{22-25}$ which deal with the temperature dependence of Raman scattering from carbon nanotubes nearly all of this literature focuses on shifts in the resonance energy and phonon energies with temperature. There are very few papers which contain any data on the temperature dependence of the strength of the scattering despite its sensitivity to coherence effects. In many papers the resonances are presented in a normalised form which prevents 
comparison of amplitudes between temperatures. ${ }^{22}$ After considerable literature searching we found one paper, Simon et al. ${ }^{25}$, which presents un-normalised RBM resonance profiles as a function of temperature but does not explicitly analyse the temperature dependence of the strength of the scattering and a second paper, May et al., ${ }^{26}$ which presents data and analyses the temperature dependence of the scattering strength. May et al. present results on metallic and semiconducting single walled carbon nanotubes in the temperature range 300K-775K. The strength of the RBM Raman scattering from the RBMs of the semiconducting tubes decreases significantly with increasing temperature in a manner similar to our results on $\mathrm{HgTe}$ nanowires. However they were unable to reliably determine the linewidth of the resonance and so were unable to link linewidth and amplitude. In addition they confuse the temperature dependence of the population lifetime determined by Perebeinos et al., ${ }^{27}$ with the coherent lifetime. Simon et al. present data on $(6,4)$ nanotubes in the form of single walled carbon nanotubes and as inner tubes within double walled nanotubes. The temperature dependence of the scattering strength and the resonance linewidth in both cases is fully in line with the results and interpretation presented here it is not explicitly analysed in the original paper. The approximate 10 fold reduction in the strength of the scattering between $80 \mathrm{~K}$ and $600 \mathrm{~K}$, can be explained by a linewidth broadening by approximately a factor of 2 and a change increase in the thermal phonon population by a factor of 1.75 (see supplementary information), which would lead to a factor of $\sim 16$ change in the strength of Raman scattering. This is in line with Simon et al.'s attribution of the increased scattering strength for $(6,4)$ tubes when surrounded by a second tube compared with when exposed directly to the environment. It interesting to note, that the measured resonance linewidth of our embedded HgTe nanotubes is very close to that of the inner tube in a double walled carbon nanotube. This suggests the possibility that the mechanism for decoherence might be associated with the surrounding tube in both cases however clearly data on other embedded wires would be required before any firm conclusion could be drawn.

\section{Conclusions}

This paper presents a full characterisation of the temperature dependence of the Raman scattering from 2 atom diameter HgTe nanowires and its resonant behaviour at laser energies in the range 1.65 to 1.90 for temperatures up to $292 \mathrm{~K}$. The data shows that the four fundamental phonon modes responsible for the Raman scattering soften and broaden with temperature as is common in crystalline materials. A detailed analysis of the excitation photon energy dependence of the Raman scattering indicates that the Raman scattering due to the $A$ and $B$ phonon modes are resonant at slightly different energies; $1.75 \mathrm{eV}$ and $1.77 \mathrm{eV}$ at $4 \mathrm{~K}$. The existence of Raman peaks due to a combination of both phonons means that the optical transitions responsible for the resonances must co-exist in the same material and the overlap in energy of the two transitions suggests they are closely related. This is supported by the fact that the resonance energies red shift with increasing temperature whilst maintaining a constant energy separation of approximately $50 \mathrm{meV}$. The linewidths of the resonances associated with different Raman peaks vary in the range $20-40 \mathrm{meV}$ at $4 \mathrm{~K}$ and in all cases increase significantly with increasing temperature. By far the largest effect of temperature on the Raman scattering from the HgTe nanowires is that the strength of the scattering at the peak of the resonance drops dramatically with increasing temperature. It drops by at least an order of magnitude for all the peaks and by a factor of 20 for the most sensitive analysable peak, the $3 \mathrm{~B}$, over the measured temperature range.

It is possible to explain the temperature dependence of the strength of the Raman scattering from the HgTe nanowires using the standard perturbation theory for Raman scattering if we assume that the linewidth of the underlying optical transition responsible for the resonance is predominantly due to temperature dependent coherence lifetime broadening. Whilst this conclusion is based solely upon data for the $\mathrm{B}, 2 \mathrm{~B}$ and $3 \mathrm{~B}$ peaks it is not contradicted by the data on the other Raman peaks and all of the peaks show the same strong temperature dependence which suggests the conclusion is also true for the other resonances. It is interesting to note that the conclusion that the optical transition responsible for the resonance of the $B$ peak and its harmonics can be treated as lifetime broadened single frequency transition is valid despite the fact that the difference between the $A$ and $B$ resonance centre energies is comparable to the resonance width. This suggests that the transitions which produce the different resonance energies couple to one or other of the $A$ and $B$ phonons and not both.

As set out in the paper it is not entirely clear from the results whether multiphonon Raman in the HgTe nanowires is due to sequential or simultaneous emission of the phonons. The temperature dependence of the scattering strength suggest that sequential emission is more likely however the relative linewidth of the different resonances suggest that simultaneous emission is more likely. It is clearly possible for both mechanisms to be active at the same time and this may explain the apparent contradiction between the two aspects of the data. Another possible explanation is that multi-phonon Raman involves additional dark intermediate states as is generally accepted to be true in the case of multiphonon Raman in bulk semiconductors \{\} .

The fact that the temperature dependence of the scattering strength can be explained across the full temperature range of the experiments by assuming that the resonance linewidth is dominated by coherence lifetime broadening strongly suggests this hypothesis is correct. If so then we can determine the coherence lifetimes at $50 \mathrm{~K}$ and $295 \mathrm{~K}$ are $18 \mathrm{fs}$ and $9 \mathrm{fs}$ respectively. It is interesting to note that these lifetimes are to within experimental error the same as the coherence lifetimes of $(6,4)$ carbon nanotubes when embedded in a second carbon nanotube. ${ }^{25}$

More generally, these results add to our understanding of coherence related effects in $1 \mathrm{D}$ quantum systems. The agreement 
between the results presented here and the two existing equivalent measurements on carbon nanotubes suggests that despite having 1D of freedom, leading to bands of energy states, the optical transitions in both systems can be treated as coherently broadened transitions with discrete energies. In the case of carbon nanotubes the discrete energies are due to excitonic transitions and there are good reasons for believing the transition responsible for the Raman resonance in the HgTe nanowires is also excitonic. Whether practical applications of the coherence of optical transitions in 1D systems can be developed is still unclear, however the optical properties of carbon nanotubes is generating a huge amount of interest $^{28,29}$ and small diameter nanowires such as the ones investigated in this paper are likely to bring additional new complementary features to this field.

In addition these results have general implications for the application of Raman scattering to nanowires. In particular these results indicate that for some nanowire systems that low temperature Raman measurements may be required in order to obtain a measurable spectrum. The lack of strong temperature dependence of Raman in 2D and 3D systems means that the practical difficulties of cryogenic sample environments are commonly not worth the effort. This may not be the case in 1D systems. Another more general point is that the temperature dependence of the intensity of Raman scattering does not receive sufficient attention. A clear example of this are the carbon nanotube results reported here which whist less eye catching contain significant physics. Whilst measuring the temperature dependence of the intensity is more challenging than measuring the temperature dependence of the other peak parameters it can be done with a reasonable accuracy with enough care and attention and can further elucidate the physics of the system being investigated.

\section{Acknowledgements}

This works was supported by EPSRC grant EP/1033394/1. J.S. and R.J.K. are indebted to the Warwick Centre for Analytical Science (EPSRC funded grant EP/F034210/1). The authors would also like to thank Dr. Simone De Liberato for his thoughts and helpful discussion on Raman theory. All correspondence should be addressed to David C. Smith (d.c.smith@soton.ac.uk).

\section{Notes and references}

M. Bockrath, D. H. Cobden, J. Lu, A. G. Rinzler, R. E. Smalley, L. Balents and P. L. McEuen, Nature, 1999, 397, 598-601. S. Nadj-Perge, I. K. Drozdov, J. Li, H. Chen, S. Jeon, J. Seo, A H. MacDonald, B. A. Bernevig and A. Yazdani, Science (80-. )., 2014, 346, 602-607. F. Wang, D. J. Cho, B. Kessler, J. Deslippe, P. J. Schuck, S. G. Louie, A. Zettl, T. F. Heinz and Y. R. Shen, Phys. Rev. Lett., 2007, 99, 227401

Lieber, Nature, 2002, 415, 617-620.

M. S. Dresselhaus, G. Dresselhaus, R. Saito and a. Jorio, Phys. Rep., 2005, 409, 47-99.

M. V. Kharlamova, Uspekhi Fiz. Nauk, 2013, 183, 11451174.

H. Kataura, Y. Kumazawa, Y. Maniwa, I. Umezu, S. Suzuki, Y. Ohtsuka and Y. Achiba, Synth. Met., 1999, 103, 2555-2558.

S. Reich, C. Thomsen and P. Ordejón, Phys. Rev. B, 2002, 65, 155411.

J. Lin, O. Cretu, W. Zhou, K. Suenaga, D. Prasai, K. I. Bolotin, N. T. Cuong, M. Otani, S. Okada, A. R. Lupini, J.-C. Idrobo, D. Caudel, A. Burger, N. J. Ghimire, J. Yan, D. G. Mandrus, S. J. Pennycook and S. T. Pantelides, Nat. Nanotechnol., 2014, 9, 436-42.

R. Senga, H.-P. Komsa, Z. Liu, K. Hirose-Takai, A. V Krasheninnikov and K. Suenaga, Nat Mater, 2014, 13, 1050-1054.

A. V Krestinin, N. A. Kiselev, A. V Raevskii and A. G. Ryabenko, Eurasian Chem.-Technol. J., 2003, 5, 7-18.

R. Carter, J. Sloan, A. Kirkland, R. Meyer, P. Lindan, G. Lin, M. Green, A. Vlandas, J. Hutchison and J. Harding, Phys. Rev. Lett., 2006, 96, 215501.

J. Sloan, D. M. Wright, S. Bailey, G. Brown, A. P. E. York, K. S. Coleman, M. L. H. Green, D. M. Wright, J. L. Hutchison and H.-G. Woo, Chem. Commun., 1999, 699-700.

G. Brown, S. R. Bailey, M. Novotny, R. Carter, E. Flahaut, K. S. Coleman, J. L. Hutchison, M. L. H. Green and J. Sloan, Appl. Phys. A Mater. Sci. Process., 2003, 76, 457-462.

D. C. Smith, J. H. Spencer, J. Sloan, L. P. McDonnell, H. Trewhitt and R. J. Kashtiban, J. Vis. Exp., 2016.

J. H. Spencer, J. M. Nesbitt, H. Trewhitt, R. J. Kashtiban, G. Bell, V. G. Ivanov, E. Faulques, J. Sloan and D. C. Smith, ACS Nano, 2014, 8, 9044-9052.

R. Carter, J. Sloan, A. I. Kirkland, R. R. Meyer, P. J. D. Lindan, G. Lin, M. L. H. Green, A. Vlandas, J. L. Hutchison and J. Harding, Phys. Rev. Lett., 2006, 96, 215501.

N. Kuganathan and J. Green, Int. J. Quantum Chem., 2008, 807, 797-807.

A. Yariv, IEEE J. Quantum Electron., 1977, 13.

I. B. Mortimer and R. J. Nicholas, Phys. Rev. Lett., 2007, 98, 1-4.

F. Wang, G. Dukovic, L. Brus and T. F. Heinz, Science (80-. )., 2011, 838.

S. B. Cronin, Y. Yin, A. Walsh, R. B. Capaz, A. Stolyarov, P. Tangney, M. L. Cohen, S. G. Louie, A. K. Swan, M. S. Ünlü, B. B. Goldberg and M. Tinkham, Phys. Rev. Lett., 2006, 96, 2225.

C. Fantini, A. Jorios, M. Souza, M. S. Strano, M. S. Dresselhaus and M. a. Pimenta, Phys. Rev. Lett., 2004, 93, $1-4$.

A. Jorio, C. Fantini, M. Dantas, M. Pimenta, A. Souza Filho, G. Samsonidze, V. Brar, G. Dresselhaus, M. Dresselhaus, A. Swan, M. Ünlü, B. Goldberg and R. Saito, Phys. Rev. B, 
2002, 66, 1-8.

F. Simon, R. Pfeiffer and H. Kuzmany, Phys. Rev. B Condens. Matter Mater. Phys., 2006, 74, 1-4.

P. May, H. Telg, G. Zhong, J. Robertson, C. Thomsen and J. Maultzsch, Phys. Rev. B - Condens. Matter Mater. Phys., 2010, 82, 1-6.

V. Perebeinos, J. Tersoff and P. Avouris, Nano Lett., 2005, 5, 2495-2499. A. Sharma, V. Singh, T. L. Bougher and B. A. Cola, Nat Nano 2015, 10, 1027-1032.

M. S. Hofmann, J. T. Gluckert, J. Noe, C. Bourjau, R. Dehmel and A. Hogele, Nat. Nanotechnol., 2013, 8, 502-505. A. Werner, H. D. Hochheimer, K. Strössner and A. Jayaraman, Phys. Rev. B, 1983, 28, 3330-3334. 